\title{
A Simple, Robust Orthogonal Background Correction Method for Two-Dimensional Liquid Chromatography
}

\author{
Marcelo R. Filgueira, ${ }^{\dagger,}$ Cecilia B. Castells, ${ }^{\ddagger}$ and Peter W. Carr ${ }^{\dagger} * *$ \\ ${ }^{\dagger}$ Department of Chemistry, Smith and Kolthoff Halls, University of Minnesota, 207 Pleasant Street S.E., Minneapolis, Minnesota \\ 55455, United States \\ *Universidad Nacional de La Plata, , División Química Analítica, Facultad de Ciencias Exactas, 47 y 115, La Plata RA-1900, Argentina
}

ABSTRACT: Background correction is a very important step that must be performed before peak detection or any quantification procedure. When successful, this step greatly simplifies such procedures and enhances the accuracy of quantification. In the past, much effort has been invested to correct drifting baseline in one-dimensional chromatography. In fast online comprehensive two-dimensional liquid chromatography $(\mathrm{LC} \times \mathrm{LC})$ coupled with a diode array detector $(\mathrm{DAD})$, the change in the refractive index (RI) of the mobile phase in very fast gradients causes extremely serious baseline disturbances. The method reported here is based on the use of various existing

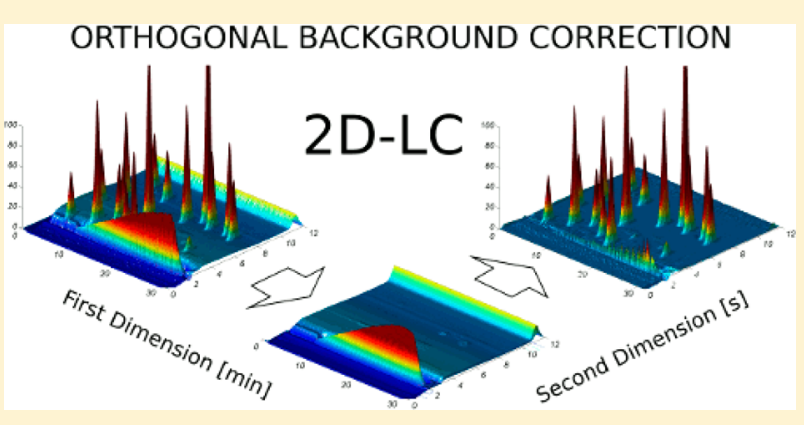
baseline correction methods of one-dimensional (1D) liquid chromatography to correct the two-dimensional (2D) background in LCXLC. When such methods are applied orthogonally to the second dimension $\left({ }^{2} \mathrm{D}\right)$, background correction is dramatically improved. The method gives an almost zero mean background level and it provides better background correction than does simple subtraction of a blank. Indeed, the method proposed does not require running a blank sample.

$\mathrm{P}$ eak detection is one of the key steps in virtually all chromatographic data analysis, especially in metabolomic studies. ${ }^{1}$ This process is greatly affected by the background signal which can show considerable variations with time. Background correction is a very important step that should be addressed prior to any quantitative analysis to reduce the difficulty of peak detection and enhance the quality of the quantitative results. ${ }^{2,3}$

It has been shown that, when a first dimension $\left({ }^{1} \mathrm{D}\right)$ peak is sampled into the ${ }^{2} \mathrm{D}$, both the peak height and area of the resulting series of ${ }^{2} \mathrm{D}$ peaks will adequately represent the ${ }^{1} \mathrm{D}$ peak, even with different sampling phases, provided that at least three samples are taken across the ${ }^{1} \mathrm{D}$ peak. ${ }^{4}$ When peaks are not fully resolved, finding the integration limits becomes more difficult. In this situation, peak height is recommended for quantitative purposes. ${ }^{5}$ Even with integration limits properly assigned, when for example an incompletely resolved small peak elutes before a larger peak, the peak height is strongly recommended over the peak area as a quantitative measurement of the smaller peak. ${ }^{6}$

The peak height, along with the height of the second derivative of the signal, can be a robust method to determine the peak width when peaks are not fully resolved. ${ }^{7}$ For this reason, correcting the baseline is important to obtain the correct peak height and peak width. If the baseline has significant drift, the peak height can be greatly affected and correcting the baseline becomes critical. The magnitude of the effect of the baseline in peak quantification can be very different depending on the shape of the baseline, on the region in the chromatogram where the peaks elute, and also on the relative height of the peak.

When background signals are not corrected, chemometric analysis can be affected because most of the variance of the data set might be due to the baseline. ${ }^{8}$ While the method developed in this work is illustrated with univariate data, it can be applied to all channels in a multivariate data set.

In this work, an orthogonal background correction (OBGC) method is developed and shown to be very useful for correcting the complex DAD background signals in fast online LC $\times \mathrm{LC}$.

\section{EXPERIMENTAL SECTION}

Two types of data were employed in this work to show and discuss the principle of the OBGC method. The first data type consists of real LCXLC chromatographic data acquired in this lab. The second data type is hybrid data created by adding simulated 2D Gaussian peaks to a series of real replicates of the background acquired by doing experimental dummy (blank) LCXLC runs.

Real Data Set. The chromatograms corresponding to online $\mathrm{LC} \times \mathrm{LC}$ runs, where gradient elution was used in both ${ }^{1} \mathrm{D}$ and ${ }^{2} \mathrm{D}$, are explained in detail in our previous work. ${ }^{9}$ A general description of the conditions is provided here:

The ${ }^{1} \mathrm{D}$ column was a Zorbax Bonus-RP $100 \mathrm{~mm}$ long by 2.1 $\mathrm{mm}$ internal diameter packed with $3.5 \mu \mathrm{m}$ particles (Agilent Technologies, Inc., Wilmington, DE). The ${ }^{1} \mathrm{D}$ eluent was $10 \mathrm{mM}$ phosphate buffer ( $\mathrm{pH}$ : 5.7) on channel A and acetonitrile on channel B. A linear gradient program was used from 0 to $24 \mathrm{~min}$, 0 to $50 \%$ of channel B. At $24.01 \mathrm{~min}$, the composition was

Received: May 8, 2012

Accepted: June 15, 2012

Published: June 15, 2012 
restored to the initial conditions. The flow rate in the column was $0.1 \mathrm{~mL} / \mathrm{min}$, and temperature was controlled at $40^{\circ} \mathrm{C}$.

The ${ }^{2} \mathrm{D}$ column was an in-house $33 \mathrm{~mm}$ long by $2.1 \mathrm{~mm}$ internal diameter packed with $3.0 \mu \mathrm{m}$ ZirChrom-CARB particles (ZirChrom Separations, Anoka, MN). The mobile phase was 10 $\mathrm{mM}$ phosphoric acid on channel A and acetonitrile on channel B. A linear gradient was used from 0 to $0.30 \mathrm{~min}, 0$ to $100 \%$ of channel B. At $0.31 \mathrm{~min}$, the gradient returned to the initial conditions allowing $3 \mathrm{~s}$ for system reequilibration, corresponding to a cycle time of $21 \mathrm{~s}$. The flow rate in the column was $3.0 \mathrm{~mL} /$ min, and the temperature was controlled at $110^{\circ} \mathrm{C}$.

A standard mixture of various indole derivatives was injected, using the same conditions as previously described. The experimental procedure for sample preparation and system configuration has been described in detail. ${ }^{9}$

Hybrid Data Set. A simple in-house program was developed using Matlab R14 (R2011b, The Mathworks Inc., MA) to simulate 20 2D Gaussian peaks using the following equation:

$$
\text { signal }=\text { area } \times \exp -\frac{1}{2}\left(\left(\frac{X-{ }^{1} t_{\mathrm{R}}}{{ }^{1} \sigma}\right)^{2}+\left(\frac{Y-{ }^{2} t_{\mathrm{R}}}{{ }^{2} \sigma}\right)^{2}\right)
$$

where $X$ and $Y$ correspond to the independent variable on each dimension, ${ }^{1} t_{\mathrm{R}}$ and ${ }^{2} t_{\mathrm{R}}$ are the retention time of the ${ }^{1} \mathrm{D}$ and ${ }^{2} \mathrm{D},{ }^{1} \sigma$ and ${ }^{2} \sigma$ are the standard deviation of the Gaussian peak on the ${ }^{1} \mathrm{D}$ and ${ }^{2} \mathrm{D}$, respectively.

The retention times in both dimensions for each peak were pseudorandomly generated (uniformly distributed) using the rand() function provided in Matlab. The peak height was also pseudorandomly generated (exponentially distributed) using an average peak height of 20 . The generated $2 \mathrm{D}$ peaks were then added to the corresponding data matrix of real $\mathrm{LC} \times \mathrm{LC}$ chromatograms acquired from five replicate dummy runs. In this way, the characteristics of the peaks were perfectly known and the background was representative of real experiments.

Experimental Conditions for the Dummy Runs. Chemicals: Chromatographic grade water and acetonitrile were obtained from Fisher Scientific (Pittsburgh, PA). Reagent grade perchloric acid was purchased from Mallinkdrodt-Baker (Paris, $\mathrm{KY}$ ). All materials were used as received. All mobile phases were prepared gravimetrically $( \pm 0.01 \mathrm{~g})$ and used without any further filtration. Chromatographic conditions: The mobile phases used for both the ${ }^{1} \mathrm{D}$ and ${ }^{2} \mathrm{D}$ were $10 \mathrm{mM}$ perchloric acid in water in channel $\mathrm{A}$ and acetonitrile in channel $\mathrm{B}$. In the ${ }^{1} \mathrm{D}$, the column used was a Zorbax SB-C3 $100 \mathrm{~mm}$ long by $4.6 \mathrm{~mm}$ internal diameter, packed with $3.5 \mu \mathrm{m}$ particles (Agilent Technologies, Inc., Wilmington, DE). The gradient was $0-56 \% \mathrm{~B}$ in $0-24.5$ $\mathrm{min}, 0 \% \mathrm{~B}$ at $24.51 \mathrm{~min}$. The flow rate in the ${ }^{1} \mathrm{D}$ column was 0.5 $\mathrm{mL} / \mathrm{min}$ and the flow rate in the splitting pump was $0.1 \mathrm{~mL} /$ min. ${ }^{10}$ The column was maintained at a temperature of $40^{\circ} \mathrm{C}$. In the ${ }^{2} \mathrm{D}$, the column was the same as previously described for the ${ }^{2} \mathrm{D}$ of the Real Data set. The gradient was $0-100 \% \mathrm{~B}$ in $0-0.15$ $\min , 0 \% \mathrm{~B}$ at $0.16 \mathrm{~min}$. The cycle time was $0.2 \mathrm{~min}(12 \mathrm{~s})$. The flow rate was $3 \mathrm{~mL} / \mathrm{min}$ with the column maintained at a temperature of $110{ }^{\circ} \mathrm{C}$. The instrument configuration was the same as in a previous publication from this group, using the split mode. $^{10}$

Data Processing. All data were acquired by Agilent Chemstation software version B.04.03 (Agilent Technologies $\mathrm{GmbH}$, Waldbronn, Germany) as a single chromatogram for each LC $\times$ LC run and then exported as a comma-separated values (csv) file and processed using Matlab with in-house written programs.

Convention and Nomenclature. The terminology adopted for this work is as much as possible in agreement with the recent update by Marriot et al. ${ }^{11}$

\section{RESULTS AND DISCUSSION}

Background Structure in Fast Online LCXLC-DAD. Many authors have shown that using a gradient in both dimensions in $\mathrm{LC} \times \mathrm{LC}$ is the best way to maximize the peak capacity. ${ }^{12,13}$ When gradient elution is used, the change in mobile phase composition causes drifts in the baseline due to two different effects: (1) the difference in absorbance between the blended solvents and (2) changes in the refractive index (RI) of the mobile phase. Because the ${ }^{2} \mathrm{D}$ gradients have to be very fast (e.g., $100 \%$ in $9 \mathrm{~s}$ gradient for $12 \mathrm{~s}$ cycle time) to minimize the undersampling effect, ${ }^{14}$ the baseline is greatly affected, as shown in Figure 1 for an $18 \mathrm{~s}$ gradient with $3 \mathrm{~s}$ of reequilibration time (21 s cycle time).

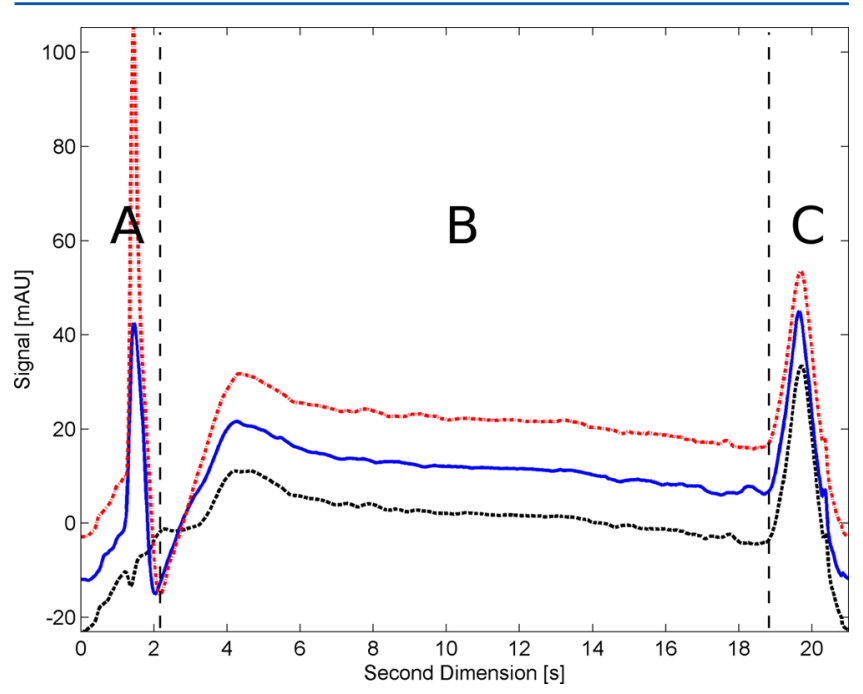

Figure 1. Structure of the LCXLC background represented by three single ${ }^{2} \mathrm{D}$ chromatograms of a dummy run sampled at different times of the gradient in the ${ }^{1} \mathrm{D}$. The amount of acetonitrile in the sample solvent transferred from the ${ }^{1} \mathrm{D}$ to the ${ }^{2} \mathrm{D}$ (region $\mathrm{A}$ ) corresponds to $10 \%$ for the black dotted curve, $30 \%$ for the blue curve, and $50 \%$ for the red dashed curve. Region $\mathrm{B}$ is where most peaks elute, and region $\mathrm{C}$ shows the system flush-out peak.

Structure of the LCXLC Background. Figure 1 shows three single ${ }^{2} \mathrm{D}$ chromatograms of an entire LC $\times$ LC run (typically about 90 single ${ }^{2} \mathrm{D}$ runs for a $30 \mathrm{~min}$ experiment). For convenience, we will divide the ${ }^{2} \mathrm{D}$ chromatograms into three different regions (A, B, and C), corresponding to three different features:

(A) In the first region ( 0 to $2 \mathrm{~s}$ ) we observe a very sharp peak caused principally by the RI mismatch of the ${ }^{1} \mathrm{D}$ eluent which delivers the sample into the initial gradient conditions of the ${ }^{2} \mathrm{D}$ (100\% aqueous). The ${ }^{1} \mathrm{D}$ gradient also starts with a $100 \%$ aqueous mobile phase with a linearly increasing volume fraction of organic solvent. As the ${ }^{2} \mathrm{D}$ always starts at $100 \%$ water, the sharp initial peak in region $\mathrm{A}$ will increase in magnitude as the chromatographic run in the ${ }^{1} \mathrm{D}$ progresses (see Figure 1). In a 3D plot of the LC $\times \mathrm{LC}$ chromatogram, this effect manifests itself as a ridge (see Figure 4). As the organic fraction of the 

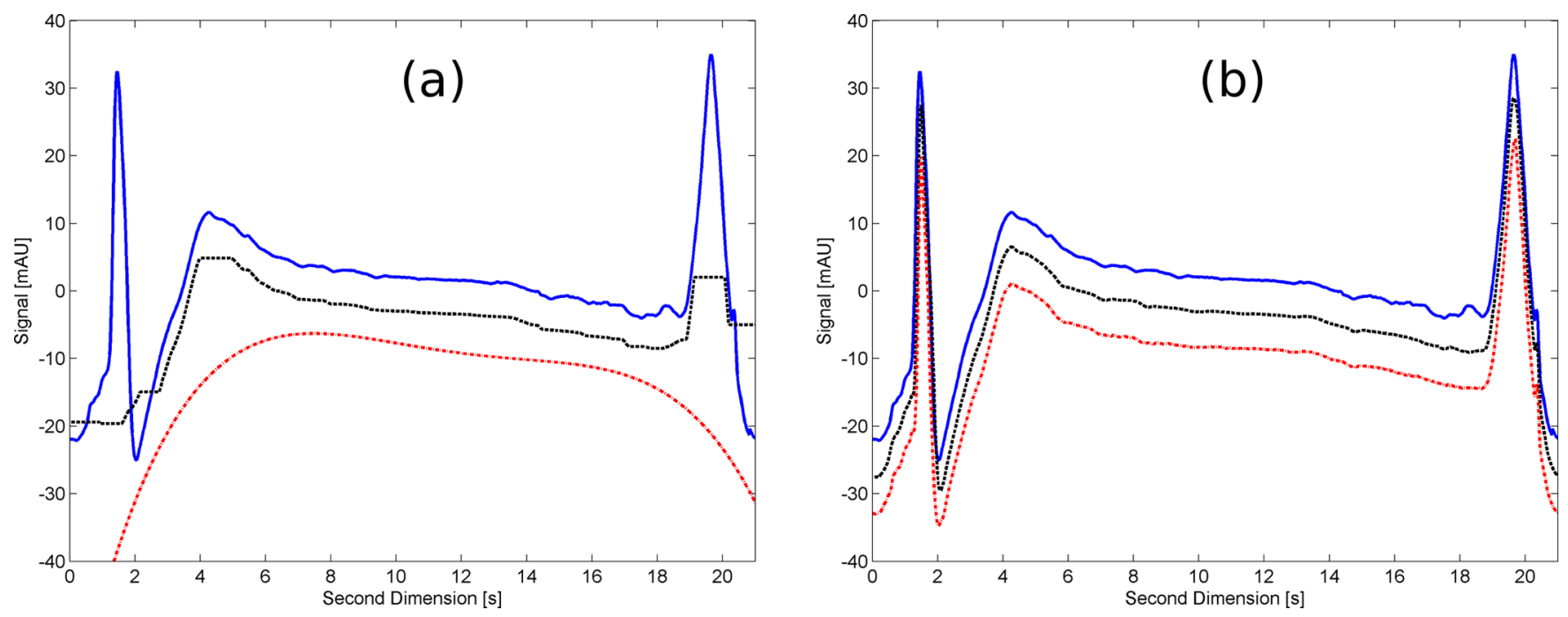

Figure 2. Comparison of estimated baselines using the different methods on a typical single ${ }^{2} \mathrm{D}$ chromatogram. The chromatograms are intentionally offset by $7 \mathrm{mAU}$ to help visualization. (a) Conventional baseline correction methods: the blue solid line chromatogram is the real single ${ }^{2} \mathrm{D}$ chromatogram; the black dashed line is the estimated baseline using the moving-median filter, and the red dot-dashed line is the estimated baseline using the polynomial fitting method. (b) The two methods are applied in combination with the OBGC method; the line format is same as in a.

sample solvent increases, so does the height of the ridge due to the RI mismatch increase. Usually only a few sample peaks elute in this region.

(B) Next is the region from 2 to $19 \mathrm{~s}$ in Figure 1, where the broad "bump" and slightly negatively going baseline is due to RI and absorbance mismatch between both channels in the ${ }^{2} \mathrm{D}$ gradient, but some very small baseline disturbances are probably due to incomplete mixing of the two solvents during these very fast gradients. Also, the first few seconds of the baseline can be affected, but the magnitude of the impact depends on the switching speed of the sampling valve, the relative volume of sample transferred to the ${ }^{2} \mathrm{D}$, and the solvent's composition. We find that the faster the valve switches, the less the baseline is perturbed. This is the region in which most sample peaks elute.

(C) The last region, from 19 to $21 \mathrm{~s}$, shows a broader peak of essentially fixed height which is caused by the system "flush-out" ( ${ }^{2} \mathrm{D}$ pump's mixer, tubing, and column) when the gradient rapidly returns to $100 \%$ aqueous mobile phase after completing each ${ }^{2} \mathrm{D}$ gradient. This again is principally due to RI mismatch, but because the mobile phase passes through the ${ }^{2} \mathrm{D}$ pump's gradient mixer, the resulting peak is much wider than the first sharp peak in region A. Some sample peaks that occasionally do not completely elute during the gradient may elute in this region though this is not desirable.

Clearly, the baselines observed in LCXLC with dual gradients and very fast ${ }^{2} \mathrm{D}$ coupled with a DAD show large and very irregular drifts (frequently tens of mAUs), and these baseline disturbances must be removed (subtracted) from the individual ${ }^{2} \mathrm{D}$ chromatograms when a real sample is analyzed.

As noted above, the signal taken in the direction of the ${ }^{1} \mathrm{D}$ is expected to be very reproducible across the large number of ${ }^{2} \mathrm{D}$ chromatograms that comprise a single LC $\times$ LC chromatogram; the ${ }^{1} \mathrm{D}$ signal varies only slowly, if at all, except in region $\mathrm{A}$ where the ${ }^{1} \mathrm{D}$ injection solvent peak exists (see Figure 1, region $\mathrm{A}$ ). This slow change across the ${ }^{1} \mathrm{D}$ is the key characteristic underlying the OBGC method.
Many different methods for baseline correction in chromatography and spectroscopy have been developed for use with univariate and multivariate data. It is beyond the scope of this work to review and test all of them. ${ }^{8,15-18}$

Two popular baseline correction methods are used here to show the principle of the OBGC approach. One is the movingmedian filter applied to separation science for the first time by Moore and Jorgenson in $1993 .{ }^{19}$ In this method a median filter is applied as a moving window, where the window has to be, at least, twice the width of the signal peaks to properly "filter" the data; in a second step, the filtered signal is subtracted from the original chromatogram to correct the baseline. As they point out in their article, "it works best when the peaks of interest are on a very different time scale from the undesirable background". The criterion of using a filter window size that is at least twice the width of the widest peak was used in this work. The median filter was implemented by means of the medfilt2() function in Matlab. The other baseline correction method used here was proposed by Mazet et al. $^{20}$ They applied an explicit asymmetric cost function where the baseline is fitted by a polynomial, which is subtracted from the original signal in a second step (asymmetric polynomial fitting). The Matlab code of their method was graciously shared by the authors under the BSD license, and it is available online at Matlab Central. ${ }^{21}$ In our experience, polynomial orders no higher than five provided the best fitting to the data, although the specific parameters of the method must be tailored to the data to provide the best results.

When we consider the baseline of a single ${ }^{2} \mathrm{D}$ chromatogram with the structure shown in Figure 1, we found that no single method could effectively fit the background structure as shown in Figure 2a.

While the filter parameters used could probably be improved, clearly neither method is able to precisely reproduce the baseline and thus subtracting it from a sample bearing chromatogram will leave a lot of extraneous background. In the case of the movingmedian filter, if a narrower window would have been used, real peaks would also have been removed from the chromatogram. With the asymmetric polynomial fitting method, we were not able to mimic the background by using higher order polynomials. 

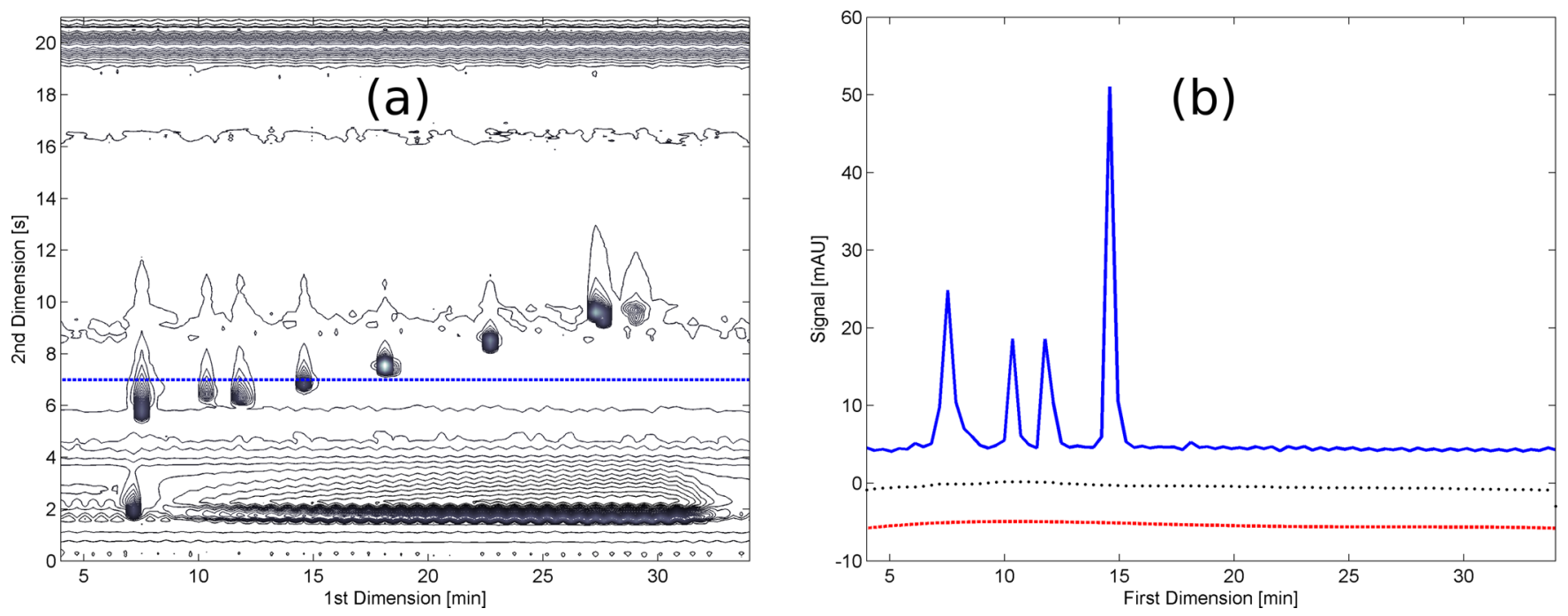

Figure 3. (a) Contour plot of a LCXLC chromatogram of an indole standard sample; the blue dotted line shows a cut taken at $7.0 \mathrm{~s}$ in the ${ }^{2} \mathrm{D}$. (b) Blue curve: chromatogram taken as the ${ }^{1} \mathrm{D}$ cut represented by the blue dotted line in panel a; black dotted curve: baseline generated by the moving-median filter; red dashed curve: baseline resulting from the asymmetric polynomial fitting.

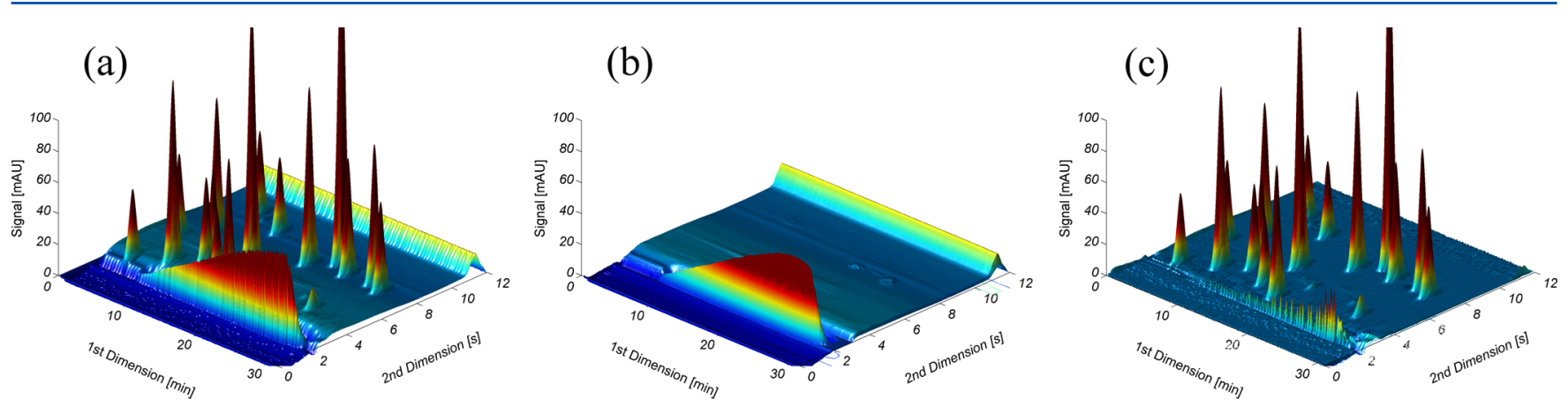

Figure 4. $3 \mathrm{D}$ plots of the OBGC method represented as (a) the original hybrid chromatogram of 20 simulated $2 \mathrm{D}$ peaks over a dummy LC $\times \mathrm{LC}$ chromatogram, (b) the recreated background structure obtained applying the OBGC method in combination with the moving-median filter, and (c) the result of subtracting the recreated background shown in $\mathrm{b}$ to the original hybrid chromatogram shown in a.

To understand how the OBGC is applied, consider the contour plot shown in Figure 3a. If we take the cut shown as the blue dashed line and plot the intensities vs ${ }^{1} \mathrm{D}$ time, this will represent a ${ }^{1} \mathrm{D}$ chromatogram, sampled at a time of $7.0 \mathrm{~s}$ of the ${ }^{2} \mathrm{D}$, as shown in Figure $3 \mathrm{~b}$. This process has to be repeated for every ${ }^{2} \mathrm{D}$ time point, which for a $21 \mathrm{~s}$ cycle time using an acquisition rate of $80 \mathrm{~Hz}$ in the detector will result in $1680^{1} \mathrm{D}$ chromatograms that will recreate the complete LCXLC background. Figure $2 \mathrm{~b}$ shows the corrected baseline with the OBGC method for the same baseline as in Figure 2a. This way, the OBGC method is also applied in two steps as the two conventional methods:

(1) Apply a specific conventional method across the ${ }^{1} \mathrm{D}$ direction to each ${ }^{2} \mathrm{D}$ data point to recreate the LCXLC background.

(2) Subtract the LC $\times$ LC background generated in the previous step from the original LC $\times$ LC chromatogram.

The chief advantage of this procedure is that any background effect from the sample of the ${ }^{1} \mathrm{D}$, basically "elutes" at about the dead volume of the ${ }^{2} \mathrm{D}$, leaving a very reproducible baseline from thereafter.

Comparison with the Dummy Subtraction Method. One common way for doing background correction is to run a sample, run a blank, and subtract the blank from the sample.
However, there are two problems associated with this approach. First, one must do an extra blank run to do the correction. The second and the more important problem is that the quality of the correction depends on the reproducibility of the background. The dummy run background subtraction approach is likely to be acceptable if the dummy run is acquired within a short time of the sample run, but it becomes less acceptable the longer the time interval between the sample and the dummy runs. In considering the results here, one should understand that the dummy runs used were acquired over $10 \mathrm{~h}$. The reproducibility of this approach was measured as the standard deviation of the difference between all ten possible pairwise combinations of the five dummy runs. This standard deviation $(0.26 \mathrm{mAU})$ was compared to the standard deviation of the same five dummy runs after applying the OBGC method $(0.035 \mathrm{mAU})$. It should be noted that the standard deviation was calculated for data taken over all regions (A, B, and $\mathrm{C}$ in Figure 1). Clearly, the reproducibility of the background obtained by the OBGC method is much better than that obtained with a simple dummy subtraction.

In real applications, we need to correct LC $\times \mathrm{LC}$ chromatograms that contain many peaks of interest. In Figure 4a a typical hybrid LC $\times$ LC chromatogram is shown, in which 20 simulated 2D Gaussian peaks were added to a real dummy LC $\times \mathrm{LC}$ chromatogram. The OBGC method was applied in combination 
Table 1. Peak Height Detection Error and Reproducibility with and without the OBGC Method

\begin{tabular}{|c|c|c|c|c|c|c|c|c|}
\hline \multicolumn{3}{|c|}{ simulated values $^{a}$} & \multicolumn{3}{|c|}{ detected without $\mathrm{OBGC}^{b}$} & \multicolumn{3}{|c|}{ detected with $\mathrm{OBGC}^{c}$} \\
\hline real height $[\mathrm{mAU}]$ & ${ }^{1} t_{\mathrm{R}}[\mathrm{min}]$ & ${ }^{2} t_{\mathrm{R}}[\mathrm{s}]$ & apparent height $[\mathrm{mAU}]^{d}$ & $\% \mathrm{RE}^{e}$ & $\% \mathrm{RSD}^{f}$ & apparent height $[\mathrm{mAU}]^{d}$ & $\% \mathrm{RE}^{e}$ & $\% \mathrm{RSD}^{f}$ \\
\hline 1.76 & 6.0 & 5.11 & 6.36 & 261 & 7.77 & 1.73 & -1.87 & 3.34 \\
\hline 2.16 & 16.0 & 7.44 & 5.38 & 148 & 6.29 & 2.14 & -1.09 & 1.71 \\
\hline 3.91 & 22.2 & 9.99 & 6.91 & 76.6 & 4.07 & 3.79 & -3.23 & 5.07 \\
\hline 4.85 & 9.8 & 4.26 & 9.54 & 97.0 & 3.54 & 4.78 & -1.25 & 0.58 \\
\hline 5.10 & 27.6 & 8.81 & 7.88 & 54.5 & 3.17 & 4.99 & -2.04 & 0.40 \\
\hline 5.83 & 9.4 & 8.05 & 8.77 & 50.4 & 4.67 & 5.80 & -0.57 & 0.86 \\
\hline 7.16 & 24.2 & 6.01 & 11.4 & 58.9 & 2.82 & 7.12 & -0.55 & 0.39 \\
\hline 7.85 & 27.2 & 9.84 & 10.8 & 37.3 & 2.43 & 7.80 & -0.58 & 0.20 \\
\hline 10.8 & 21.6 & 2.64 & 7.05 & -34.7 & 6.63 & 10.7 & -0.50 & 0.74 \\
\hline 11.2 & 18.0 & 4.74 & 15.8 & 40.9 & 2.20 & 11.2 & -0.32 & 0.22 \\
\hline 12.4 & 10.0 & 10.4 & 15.6 & 26.2 & 2.38 & 12.2 & -1.54 & 0.68 \\
\hline 12.8 & 3.2 & 9.18 & 15.8 & 23.5 & 3.75 & 12.7 & -0.52 & 0.50 \\
\hline 14.2 & 20.4 & 9.69 & 17.1 & 20.4 & 1.67 & 14.1 & -0.54 & 0.41 \\
\hline 16.1 & 15.2 & 4.69 & 20.7 & 28.5 & 1.97 & 16.1 & -0.22 & 0.44 \\
\hline 20.2 & 10.8 & 3.39 & 24.8 & 22.9 & 2.43 & 20.0 & -0.51 & 0.12 \\
\hline 22.7 & 25.2 & 9.14 & 25.6 & 12.6 & 0.93 & 22.7 & -0.24 & 0.13 \\
\hline 24.4 & 6.4 & 9.35 & 27.3 & 12.1 & 1.76 & 24.3 & -0.16 & 0.27 \\
\hline 38.5 & 18.4 & 3.00 & 38.8 & 0.76 & 0.17 & 38.5 & -0.02 & 0.98 \\
\hline 40.5 & 5.8 & 3.96 & 45.3 & 11.8 & 0.79 & 40.4 & -0.22 & 0.12 \\
\hline \multirow[t]{3}{*}{66.2} & 11.4 & 10.4 & 69.5 & 4.86 & 0.51 & 66.0 & -0.32 & 0.07 \\
\hline & & & average & 47.7 & 3.00 & 16.7 & -0.81 & 0.86 \\
\hline & & & median & 27.3 & 2.43 & 11.7 & -0.53 & 0.42 \\
\hline
\end{tabular}

${ }^{a}$ Real features of the simulated 2D peaks that were added to the five real dummy LCXLC chromatogram replicates. ${ }^{b}$ Peak detection applied without any background correction to the $2 \mathrm{D}$ chromatograms obtained by adding the simulated $2 \mathrm{D}$ peaks to each of the five real dummy LCXLC chromatogram replicates (hybrid replicates). ${ }^{c}$ Peak detection applied after OBGC using the moving-median filter to the chromatograms used in b. ${ }^{d}$ Average detected peak height in the five hybrid replicates. ${ }^{e}$ Percent relative error in the measured vs the real peak height. ${ }^{f}$ Percent relative standard deviation of the measured peak height in the five hybrid replicates.

with the moving-median filter, and the recreated background is shown in Figure $4 \mathrm{~b}$. It is evident that the ridge in region A (see Figure 1) is not exactly reproduced, but the use of a smaller window for the moving-median filter results in the removal of some nonresolved peaks across the ${ }^{1} \mathrm{D}$ axes. In Figure 4, subtracting the recreated background in $\mathrm{b}$ from a gives the corrected LCXLC chromatogram in c. It is evident in Figure 4c that a very large percentage of the background is removed; however, despite the evident power of the OBGC method to remove background, some of the sample solvent ridge remains. The highly reproducible end of the gradient in region $C$ (see Figure 1) is virtually obliterated.

To measure how the background affects the measured peak heights and their reproducibility when the OBGC method is applied, the same set of 20 simulated $2 \mathrm{D}$ peaks was added to five replicate real dummy LC $\times$ LC runs. Peaks were detected and peak height was measured for both OBGC -corrected and nonbackground-corrected chromatograms. When no background correction is applied, the heights of the smaller peaks are greatly affected by the baseline as shown in Table 1 . The average error without background correction is $3.07 \pm 1.9 \mathrm{mAU}$, and while this seems to be a reasonably small number, it decreased to $-0.07 \pm$ $0.054 \mathrm{mAU}$ (a factor of 40) upon applying the OBGC method. When the background is not corrected, the percent relative standard deviation (\% RSD) of the peak heights is a measure of the reproducibility of the LCXLC instrument, because the peaks are simulated and are exactly the same in each replicate dummy LCXLC chromatogram. The average reproducibility of the corrected chromatogram is improved by a factor of 3.5 , as can be inferred from the corresponding \% RSD in Table 1.
Also, when considering long-term reproducibility (even for the same instrument), the ability to remove the background plays an important role in facilitating comparison of results. The result is a simple, more accurate, and reproducible quantification procedure.

The method described here has been put into practice with real (nonsynthetic) chromatograms. We have seen no problems other than the need to adjust the parameters of the specific function (polynomial fit, median-moving filter, etc.) used to separate the peaks from the background.

While the fast gradients will have less impact in baselines obtained with other types of detectors such as mass sensitive detectors, the high reproducibility, insensitivity to coeluent, ability to handle very high flow rates and low initial cost and maintenance, makes the $\mathrm{DAD}$ a very useful detector for fast online LCXLC.

\section{CONCLUSIONS}

The OBGC method is a very effective background correction method for LCXLC when used conjointly with currently existing baseline correction methods. The requirement of the current methods, that changes in the background be slow relative to the width of the real peaks, is readily achieved in LCXLC by use of OBGC. The OBGC method should be useful with any 2D technique wherein the ${ }^{1} \mathrm{D}$ has lower frequency baseline fluctuations than the ${ }^{2} \mathrm{D}$. Reproducibility of the peak height of measured peaks was significantly enhanced by applying the OBGC method because the system variability reflected in the background was greatly reduced, leaving an almost zero-mean background. After use of the OBGC method, the standard 
deviation corresponding to the average background noise was reduced to about $0.05 \mathrm{mAU}$.

\section{AUTHOR INFORMATION}

\section{Corresponding Author}

*Tel.: (612) 624-0253. Fax: (612) 626-7541. E-mail: petecarr@ umn.edu.

\section{Notes}

The authors declare no competing financial interest.

\section{ACKNOWLEDGMENTS}

This work was financially supported by grants from $\mathrm{NIH}$ (GM054585-15) and from NSF (CHE-0911330). We also acknowledge funding from the Agilent Foundation and the gifts of columns from Agilent Technologies Inc. and ZirChrom Separations Inc. M.F. also acknowledges a fellowship from ANPCyT-UNLP (Argentina).

\section{REFERENCES}

(1) Bailey, H. P.; Rutan, S. C.; Carr, P. W. J. Chromatogr., A 2011, 1218, $8411-8422$.

(2) Zhu, L.; Brereton, R. G.; Thompson, D. R.; Hopkins, P. L.; Escott, R. E. A. Anal. Chim. Acta 2007, 584, 370-378.

(3) Danielsson, R.; Bylund, D.; Markides, K. Anal. Chim. Acta 2002, 454, 167-184.

(4) Amador-Muñoz, O.; Marriott, P. J. Chromatogr., A 2007, 1184, 323-340.

(5) Snyder, L. R. J. Chromatogr. Sci. 1972, 10, 200-212.

(6) Felinger, A. in Data Analysis and Signal Processing in Chromatography; Elsevier Science B.V.: Amsterdam, 1998.

(7) Lan, K.; Jorgenson, J. W. Anal. Chem. 1999, 71, 709-714.

(8) Komsta, ł. Chromatographia 2011, 73, 721-731.

(9) Gu, H.; Huang, Y.; Filgueira, M.; Carr, P. W. J. Chromatogr., A 2011 , 1218, 6675-6687.

(10) Filgueira, M. R.; Huang, Y.; Witt, K.; Castells, C.; Carr, P. W. Anal. Chem. 2011, 83, 9531-9539.

(11) Marriot, P.; Schoenmakers, P.; Wu, Z. Nomenclature and conventions in comprehensive multidimensional chromatography - an update, LC-GC Europe [P], Vol. 25, Issue 5, Advanstar Communications Inc.: USA, 2012, pp 266-275.

(12) Jandera, P.; Hájek, T.; Česla, P. J. Chromatogr., A 2011, 1218, 1995-2006.

(13) Vivó-Truyols, G.; van der Wal, S.; Schoenmakers, P. J. Anal. Chem. 2010, 82, 8525-8536.

(14) Stoll, D. R.; Li, X.; Wang, X.; Carr, P. W.; Porter, S. E. G.; Rutan, S. C. J. Chromatogr., A 2007, 1168, 3-43.

(15) Eilers, P. H. C.; Boelens, H. F. M. Leiden University Medical Centre Report, 2005. http://www.science.uva.nl/ hboelens/publications/ draftpub/Eilers_2005.pdf. Acccessed on Nov 2011.

(16) Goicoechea, H. C.; Culzoni, M. J.; García, M. D. G.; Galera, M. M. Talanta 2011, 83, 1098-1107.

(17) Reichenbach, S. E.; Carr, P. W.; Stoll, D. R.; Tao, Q. J. Chromatogr., A 2009, 1216, 3458-3466.

(18) Reichenbach, S. E.; Tian, X.; Tao, Q.; Stoll, D. R.; Carr, P. W. J. Sep. Sci. 2010, 33, 1365-1374.

(19) Moore, A. W., Jr.; Jorgenson, J. W. Anal. Chem. 1993, 65 (18), $188-191$.

(20) Mazet, V.; Carteret, C.; Brie, D.; Idier, J.; Humbert, B. Chemom. Intell. Lab. Syst. 2005, 76, 121-133.

(21) Background correction - File Exchange - MATLAB Central. http://www.mathworks.com/matlabcentral/fileexchange/27429background-correction. Accessed April 2011. 